Annales Geophysicae (2002) 20: 41-55 C European Geophysical Society 2002

(5)

\title{
Effects of multiple scatter on the propagation and absorption of electromagnetic waves in a field-aligned-striated cold magneto-plasma: implications for ionospheric modification experiments
}

\author{
T. R. Robinson \\ University of Leicester, Leicester, LE1 7RH, UK \\ Received: 14 August 2000 - Revised: 8 June 2001 - Accepted: 12 June 2001
}

\begin{abstract}
A new theory of the propagation of low power electromagnetic test waves through the upper-hybrid resonance layer in the presence of magnetic field-aligned plasma density striations, which includes the effects of multiple scatter, is presented. The case of sinusoidal striations in a cold magnetoplasma is treated rigorously and then extended, in an approximate manner, to the broad-band striation spectrum and warm plasma cases. In contrast to previous, single scatter theories, it is found that the interaction layer is much broader than the wavelength of the test wave. This is due to the combined electric fields of the scattered waves becoming localised on the contour of a fixed plasma density, which corresponds to a constant value for the local upper-hybrid resonance frequency over the whole interaction region. The results are applied to the calculation of the refractive index of an ordinary mode test wave during modification experiments in the ionospheric F-region. Although strong anomalous absorption arises, no new cutoffs occur at the upper-hybrid resonance, so that in contrast to the predictions of previous single scatter theories, no additional reflections occur there. These results are consistent with observations made during ionospheric modification experiments at Troms $\emptyset$, Norway.
\end{abstract}

Key words. Ionosphere (active experiments; ionospheric irregularities) Radio science (ionospheric propagation)

\section{Introduction}

One of the most frequently observed and consistently characterised phenomena in experiments which modify the ionospheric F-region by means of high power radio waves is the anomalous absorption of low power test waves that traverse the heated volume. This effect was first discovered in ionospheric modification (heating) experiments at Platteville (Cohen and Whitehead, 1970). Soon after being reported, anomalous absorption was explained in terms of the scattering of electromagnetic waves into high frequency electro-

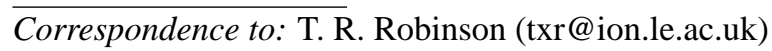

static waves at the upper-hybrid resonance, due to the presence of small-scale plasma density irregularities (Graham and Fejer, 1976; Vaskov and Gurevich, 1976). These irregularities, which are strongly elongated along the direction of the geomagnetic field, are generated by a high power electromagnetic pump wave in the vicinity of its own upper-hybrid resonance. They have been regularly observed by coherent backscatter radars during ionospheric modification experiments (Fialer, 1974; Minkoff et al., 1974; Hedberg et al., 1983; Robinson et al., 1997). Moreover, Minkoff (1974) has shown that the spectral power of the irregularities produced in heating experiments is primarly confined to crossfield scale sizes in excess of $1 \mathrm{~m}$. This result is also consistent with the estimate of a few tens of $\mathrm{km}$ for the scale length of the irregularities along the geomagnetic field line (Jones et al., 1984), since their aspect ratio is expected to be of the order of the ratio of the electron-neutral collision frequency, $\nu_{e n}$, to the electron gyro-frequency, $\Omega$. This ratio is around a few times $10^{-4}$ in the F-region. The presence of these irregularities, together with the fact that anomalous absorption only occurs for ordinary mode (O-mode) test waves and only for overdense conditions, provides strong evidence for this field-aligned striations-upper-hybrid resonance (FASTUHR) theory. Extraordinary mode (X-mode) test waves do not suffer anomalous absorption, as expected from this theory, since their reflection point occurs below the upper-hybrid resonance height. For similar reasons, only an O-mode pump can excite short-scale field-aligned plasma density irregularities and only in overdense conditions.

In several previous papers (Graham and Fejer, 1976; Vaskov and Gurevich, 1976; Dysthe et al., 1983; Jones et al., 1984), calculations of the level of anomalous absorption of a low power test wave have been carried out using the FASTUHR theory by straightforward energy considerations. This involves equating the vertical decay of the Poynting flux of the incident electromagnetic wave with the Joule loss rate per unit volume due to scattering into electrostatic waves. Furthermore, in these previous calculations, it was assumed that the propagation of the electromagnetic wave was unaffected 
by the striations. This essentially means that changes in the real part of the refractive index of the test wave, due to the presence of the striations, were neglected, whilst those in the imaginary part were not. Hagfors (1984) was the first to point out the inconsistency of this assumption and showed that, in fact, there was necessarily a strong change in the real part of the refractive index which lead to a new cutoff frequency at the upper-hybrid resonance. This cutoff, in principle, causes reflection at the upper-hybrid resonance. Indeed, Mjølhus (1985), using a formulation similar to that of Hagfors (1984), demonstrated that the anomalous absorption level could not exceed approximately $50 \%$ with the other $50 \%$ of the incident energy being reflected.

A further inconsistency in the anomalous absorption calculations of Graham and Fejer, (1976), Vaskov and Gurevich (1976), Dysthe et al. (1983) and Jones et al. (1984) is that a WKB approximation is used to integrate the loss rate across the resonance layer. The problem here is that the width of the upper-hybrid layer for exactly field-aligned irregularities is of the order of $\left(v_{e n} / \omega\right) H$, where $\omega / 2 \pi$ is the test wave frequency and $H$ is the scale height of the background electron density. For typical F-region conditions, at altitudes of around $200 \mathrm{~km}$, this layer is only a metre or so wide. This is much smaller than the wavelengths of the test waves, which are typically several tens of metres. Under these conditions the WKB approximation is invalid. It is, therefore, rather surprising that values of anomalous absorption levels obtained on the basis of the apparently inconsistent calculations of Graham and Fejer, (1976), Vaskov and Gurevich (1976), Dysthe et al. (1983) and Jones et al. (1984) agree well with the observations, whilst those of Mjølhus (1985) do not. For example, levels of anomalous absorption observed at the EISCAT high power facility at Troms $\varnothing$ can exceed $15 \mathrm{~dB}$ (Stubbe et al., 1982), which are clearly well beyond the upper limit of around $3 \mathrm{~dB}$ predicted by Mjølhus (1985). On the basis of their calculation, Jones et al. (1984) found that these levels could be obtained with striation amplitudes, indicated by the fractional deviation in electron density, $\delta$, of around $1.5 \%$. These striation amplitudes are also consistent with observed backscatter radar cross sections (Minkoff, 1974).

The source of the difficulty is not too hard to find. All of the above papers that deal with the FASTUHR theory effectively assume a weak scatter process, so that the test wave is scattered by a single Fourier component of the striations into a single electrostatic mode. In the cold plasma approximation, this electrostatic mode is non-propagating. Even under warm plasma conditions, the high frequency electrostatic wave propagates only at the electron thermal speed which is typically three orders of magnitude below the characteristic speed of the electromagnetic wave in the ionospheric Fregion. Thus, wave energy piles up in the upper-hybrid layer such that the electrostatic wave field is of the order of $\delta \omega / v_{\text {en }}$ times the incident test wave electric field. This factor can be much greater than unity under F-region conditions, which leads to a significant distortion of the refractive index of the test wave in the vicinity of the upper-hybrid layer. Both the real and imaginary parts are affected and hence, the possibil- ity of new cutoffs, as demonstrated by Hagfors (1984). However, an electrostatic wave with an amplitude larger than the original test wave can itself efficiently scatter into a further high frequency electrostatic mode. When $\delta \omega / \nu_{e n}$ is much larger than unity, this process can repeat, in principle, ad infinitum. Inhester et al. (1981) were the first to recognise the importance of higher order scatter of this type in the instability process by which the short-scale field-aligned striations are generated in the first place. Below, a new theory of anomalous absorption of a low power test wave by the FASTUHR process is developed, which completely takes into account scatter to effectively infinite order in a cold plasma. It turns out that the higher order scatter has a remarkable effect on the nature of the resonance region. Instead of being only a few metres wide, as predicted by the previous theories mentioned above, it becomes many hundreds of metres wide and, therefore, is much broader than the wavelength of a typical test wave. Furthermore, the change in the refractive index in the vicinity of the upper-hybrid resonance is now only of the order of $\delta$. With values of $\delta$ typically less than 0.05 , this has a negligible effect on the real part of the refractive index of the test wave and hence, there are, in fact, no new cutoffs. However, the change to the imaginary part of the refractive index is of the same order and this does lead to significant absorption. Remarkably, this new calculation predicts levels of anomalous absorption that are virtually the same as those predicted by the apparently inconsistent, single order scatter versions of the FASTUHR theory, cited above. Vaskov (1988) has suggested that thermal effects are sufficient to broaden the resonance region and can alleviate some of the inconsistencies indicated above. However, as will be demonstrated below, the resonance broadening due to higher order scatter, even in a cold plasma approximation, is greater than that due to thermal effects. The physical reasons for the broadening due to higher order scatter, which will also be dealt with below, are particularly interesting. They are associated with the localisation of the combined electric fields of the scattered waves. This leads to a remarkably simple interpretation in which the interaction layer is actually distorted in such a way that it follows the contours of the constant local upper-hybrid frequency. Indeed, it is the localisation effect in the cold plasma, rather than thermal effects, that ultimately limits the width of the resonance.

Before developing an expression for the complex refractive index of electromagnetic waves due to FASTUHR with multiple scattering effects in Sect. 3 below, the properties of cold plasma waves are briefly reviewed in Sect. 2. The purpose of this review is to clarify the physical processes that need to be considered when coupling is introduced between the electrostatic and electromagnetic waves. Anomalous absorption and propagation calculations appropriate to ionospheric modification experiments will be presented in Sect. 4. A discussion of the physical basis of the new theory, together with certain thermal effects will be treated in Sects. 5 and 6 , respectively.

It should be stressed that only the effects on low power test waves are considered in the present paper and the striations 
are regarded as pre-existing features which are not affected by the test wave. The case of a monochromatic $k$-spectrum of striations will be treated rigorously and then the broad-band case will be dealt with by extending the monochromatic result in an approximate way. The related issue of the generation of the striations by a high power electromagnetic pump wave will also be touched on briefly, but a detailed discussion of this important topic is beyond the scope of the present paper and will be treated in a future publication. Finally, the topic of anomalous absorption in the vicinity of harmonics of the electron gyro-frequency will not be treated in this paper, since it is strictly beyond the scope of cold plasma theory. It will, therefore, be assumed that the present theory applies only to test waves with frequencies which are not close to the harmonics of the electron gyro-frequency.

\section{Electromagnetic waves and the upper-hybrid reso- nance}

The purpose of this section is to review the dispersive properties and field equations for electromagnetic and electrostatic modes in a uniform cold magnetoplasma. Strictly speaking, there are no propagating electrostatic waves in a cold plasma. Instead, they correspond to resonances in which the refractive index tends towards infinity (Stix, 1992). However, these oscillations do have well-defined wavevectors and can be treated as longitudinal waves propagating at speeds much smaller than the speed of light. This approach, which follows Hagfors (1984) and Mjølhus (1985), makes it possible to establish the relationship between the electric field and electric current oscillations for the two modes. This facilitates a straightforward and clear extension to the coupled mode case, in the presence of irregularities in the plasma density. In addition, in what follows, only electron dynamics needs to be considered and ion motion is neglected.

The usual Maxwell equations for oscillations in the electromagnetic fields and currents of the form $\exp [i(\boldsymbol{k} \cdot \boldsymbol{x}-\omega t)]$ lead to the relation

$$
\begin{gathered}
n^{2}\left(\boldsymbol{E}(\boldsymbol{k}, \omega)-\boldsymbol{k}_{1} \boldsymbol{k}_{1} \cdot \boldsymbol{E}(\boldsymbol{k}, \omega)\right)-\boldsymbol{E}(\boldsymbol{k}, \omega) \\
=\frac{i \mu_{0} c^{2}}{\omega} \boldsymbol{J}(\boldsymbol{k}, \omega)
\end{gathered}
$$

where $n, \boldsymbol{E}(\boldsymbol{k}, \omega)$ and $\boldsymbol{J}(\boldsymbol{k}, \omega)$ are the general refractive index, electric field and current density, respectively. Also in Eq. (1), $\mu_{0}$ is the permeability of free space, $c$ is the speed of light, $\omega$ and $\boldsymbol{k}$ are the wave frequency and wavevector, respectively, and $\boldsymbol{k}_{1}$ is a unit vector along $\boldsymbol{k}$. It is assumed in what follows that the unstriated background plasma is only weakly inhomogeneous and has a small uniform gradient with a characteristic scale length that is much larger than the wavelength of any wave present. Thus, in the absence of plasma density striations, the only contribution to the current density is

$\boldsymbol{J}(\boldsymbol{k}, \omega)=-N_{0} e \boldsymbol{V}(\boldsymbol{k}, \omega)$ where $N_{0}$ is the background electron density, $e$ is the size of the electronic charge and $\boldsymbol{V}(\boldsymbol{k}, \omega)$ represents the electron velocity. In the presence of a background magnetic field and collisions between electrons and neutrals, $\boldsymbol{V}(\boldsymbol{k}, \omega)$ is given by

$$
\begin{gathered}
\boldsymbol{V}(\boldsymbol{k}, \omega)=-\frac{i}{(1+i Z)^{2}-Y^{2}}\left(\frac{(1+i Z) Y}{B_{0}} \boldsymbol{E}(\boldsymbol{k}, \omega)\right. \\
\left.-\frac{Y^{3}}{(1+i Z) B_{0}} \boldsymbol{b}_{1} \boldsymbol{b}_{1} \cdot \boldsymbol{E}(\boldsymbol{k}, \omega)+\frac{i Y^{2}}{B_{0}} \boldsymbol{b}_{1} \wedge \boldsymbol{E}(\boldsymbol{k}, \omega)\right)
\end{gathered}
$$

where $Y=\Omega / \omega, Z=v_{e n} / \omega$ and, $B_{0}$ is the flux density of the background geomagnetic field, and $\boldsymbol{b}_{1}$ is a unit vector in the geomagnetic field direction. It is important to notice that in the cold plasma approximation, $\boldsymbol{V}(\boldsymbol{k}, \omega)$ is always independent of the plasma density. It is just proportional to the electric field strength and contains no nonlinear components (unless the electron particle velocities approach the speed of light, which they do not in the present low power test wave case).

Substituting Eq. (3) into (2) and then (2) into (1) yields

$(\boldsymbol{\Pi}+\boldsymbol{\Sigma}) \boldsymbol{E}(\boldsymbol{k}, \omega)=0$

where $\Pi$ and $\boldsymbol{\Sigma}$ are matrices of the form

$$
\begin{aligned}
& \boldsymbol{\Pi}=\left(\begin{array}{ccc}
n^{2} \cos ^{2} \theta-1 & 0 & -n^{2} \cos \theta \sin \theta \\
0 & n^{2}-1 & 0 \\
-n^{2} \cos \theta \sin \theta & 0 & n^{2} \sin ^{2} \theta-1
\end{array}\right) \\
& \boldsymbol{\Sigma}=\frac{X}{(1+i Z)^{2}-Y^{2}}\left(\begin{array}{ccc}
1+i Z & -i Y & 0 \\
i Y & 1+i Z & 0 \\
0 & 0 & \frac{(1+i Z)^{2}-Y^{2}}{1+i Z}
\end{array}\right)
\end{aligned}
$$

and where $X=\left(\omega_{e} / \omega\right)^{2}$ and $\omega_{e}$ is the electron plasma frequency. In what follows, the coordinate system will always be chosen so that the $z$-axis is along $\boldsymbol{b}_{1}$. In Eq. (5), $\theta$ is the angle between $\boldsymbol{k}$ and $\boldsymbol{b}_{1}$. Thus far, the direction of $\boldsymbol{k}$ and consequently the value of $\theta$ is arbitrary. Furthermore, the direction of $\boldsymbol{k}$ will, in general, vary as the wave propagates through the inohogeneous plasma. However, the geometry for a typical ionospheric modification experiment under consideration below involves only vertical propagation of electromagnetic test waves in a horizontally stratified plasma. This case is adequately handled by choosing $\boldsymbol{k}$ for the electromagnetic modes (but not the electrostatic ones, see below) to be parallel to the weak background density gradient. This means that the direction of $\boldsymbol{k}$ and the value of $\theta$ may be taken as constant over the inhomogeneous plasma layers. This greatly simplifies the propagation calculations in Sect. 4 below, where $\boldsymbol{k}$ is chosen to lie in the $x-z$ plane for all electromagnetic modes considered.

The matrix Eq. (4) then has the explicit form

$$
\left(\begin{array}{ccc}
n^{2} \cos ^{2} \theta-S & -i D & -n^{2} \cos \theta \sin \theta \\
i D & n^{2}-S & 0 \\
-n^{2} \cos \theta \sin \theta & 0 & n^{2} \sin ^{2} \theta-P
\end{array}\right) \cdot\left(\begin{array}{l}
E_{x} \\
E_{y} \\
E_{z}
\end{array}\right)=0
$$


where

$$
\begin{aligned}
& S=1-\frac{X(1+i Z)}{(1-i Z)^{2}-Y^{2}} \\
& D=\frac{X Y}{(1+i Z)^{2}-Y^{2}} \\
& P=1-\frac{X}{1+i Z}
\end{aligned}
$$

The column vector which follows the $3 \times 3$ matrix in Eq. (7) represents the cartesian components of the electric field of the wave. To obtain the dispersion relation for the waves, the determinant of the $3 \times 3$ matrix in Eq. (7) is set to equal zero, which yields the well-known expression

$n=\sqrt{\frac{B \pm \sqrt{B^{2}-4 A C}}{2 A}}$

where

$$
\begin{aligned}
& A=S \sin ^{2} \theta+P \cos ^{2} \theta \\
& B=\left(S^{2}-D^{2}\right) \sin ^{2} \theta+P S\left(\cos ^{2} \theta+1\right) \\
& C=P\left(S^{2}-D^{2}\right)
\end{aligned}
$$

The electromagnetic modes of interest correspond to solutions of Eq. (8) in which the condition $0 \leq|n| \leq 1$ is satisfied. The positive sign option in Eq. (8) is associated with the O-mode and the negative option with the X-mode.

The properties of electrostatic waves in cold plasma can also be obtained from the above expressions. In warm plasmas, the characteristic propagation speed of high frequency electrostatic waves is the electron thermal speed. In the cold plasma approximation, this characteristic speed necessarily tends towards zero. Thus, in this approximation, electrostatic waves correspond to the resonance limit, $n \rightarrow \infty$. From Eq. (1), this implies that

$\boldsymbol{k} \wedge \boldsymbol{k} \wedge \boldsymbol{E}(\boldsymbol{k}, \omega)=0$

and hence, that $\boldsymbol{E}(\boldsymbol{k}, \omega)$ lies in the direction of $\boldsymbol{k}$. Thus,

$\boldsymbol{E}(\boldsymbol{k}, \omega)=E(\boldsymbol{k}, \omega) \boldsymbol{k}_{1}$

where $\boldsymbol{k}_{1}$ is a unit vector along $\boldsymbol{k}$. Furthermore, the scalar product of $\boldsymbol{k}_{1}$ with Eq. (1) yields the field equation for the electrostatic oscillations, which takes the form

$-E(\boldsymbol{k}, \omega)-\frac{i \mu_{0} c^{2}}{\omega} \boldsymbol{k}_{1} \cdot \boldsymbol{J}(\boldsymbol{k}, \omega)=0$

In matrix form, Eq. (12) is

$\boldsymbol{k}_{1}(-\mathbf{I}+\boldsymbol{\Sigma}) \boldsymbol{k}_{1} E(\boldsymbol{k}, \omega)=0$

where I is the unit matrix.

A further necessary condition in the resonance limit is $A=$ 0, which from Eq. (9) yields

$$
X=(1+i Z) \frac{(1+i Z)^{2}-Y^{2}}{(1+i Z)^{2}-Y^{2} \cos ^{2} \theta}
$$

In what follows, the main interest will be in electrostatic waves that have wavevectors orthogonal to the background magnetic field and hence, $\theta=\pi / 2$. This requires $S=0$ and then

$X=\frac{(1+i Z)^{2}-Y^{2}}{(1+i Z)}$

The expression in Eq. (15) corresponds to the upper-hybrid resonance in the collisional case.

\section{Effects of field-aligned plasma striations}

\subsection{The coupled equations}

In the following, it is assumed that an electromagnetic wave with an electric field $\boldsymbol{E}(\boldsymbol{m}, \omega)$ and wavevector $\boldsymbol{m}$ propagates vertically at an angle $\theta$ to the background magnetic field. Since the wavevector of all electromagnetic modes has been assumed to lie in the $x-z$ plane, this implies that the $x$ axis lies in the vertical plane, orthogonal to the geomagnetic field. The electromagnetic wave gives rise to an electron velocity, $\boldsymbol{V}(\boldsymbol{m}, \omega)$, in the plasma. It is assumed that there is a preexisting set of plane standing monochromatic plasma density striations with electron densities of the form $N(s, 0)+N(-s, 0)$, in addition to the background density. $\pm s$ are the wavevectors of the striations and are assumed to be orthogonal to the background magnetic field. The zeros in the second element of the argument in the striation density indicates that they have zero frequency in the frame of the background plasma. The presence of the striations leads to new oscillating current densities in addition to those represented by Eq. (2) above. These currents constitute two sidebands in the $k$-space which, in a weakly inhomogeneous background plasma density, take the form

$\boldsymbol{J}(\boldsymbol{m} \pm \boldsymbol{s}, \omega)=-N( \pm \boldsymbol{s}, 0) e \boldsymbol{V}(\boldsymbol{m}, \omega)$

Now these currents can be inserted as additional terms in the field equations of the type expressed in Eq. (13), for a pair of high frequency electrostatic waves with electric fields $\boldsymbol{E}(\boldsymbol{m} \pm \boldsymbol{s}, \omega)$. It will be assumed in what follows that $|\boldsymbol{m}| \ll|s|$, so that these electrostatic waves will also have wave vectors parallel to $s$ and orthogonal to the background magnetic field. It will be shown below that the electrostatic fields are large in the vicinity of the upper-hybrid resonance and will, in turn, give rise to a pair of electron velocities $\boldsymbol{V}(\boldsymbol{m} \pm \boldsymbol{s}, \omega)$, which will, in turn, give rise to further higher order current density sidebands (in the $k$-space) of the form $\boldsymbol{J}(\boldsymbol{m} \pm 2 \boldsymbol{s}, \omega)$ and also $\boldsymbol{J}(\boldsymbol{m}, \omega)$. These will provide additional contributions to the currents associated with the original electromagnetic wave, as well as to two further electrostatic waves. The generation of electric field sidebands with wavevectors which are multiples of $s$ and all with frequency $\omega$ can continue in this way ad infinitum. This leads to a set of coupled equations for the electromagnetic and the electrostatic wave fields. Defining $\delta( \pm \boldsymbol{s}, 0)$ as $N( \pm \boldsymbol{s}, 0) / N_{0}$ and $\boldsymbol{s}_{1}$ 
as a unit vector along $s$ (i.e. orthogonal to the geomagnetic field), these field equations take the form

$$
\begin{aligned}
&(\boldsymbol{\Pi}+\boldsymbol{\Sigma}) \boldsymbol{E}(\boldsymbol{m}, \omega)+\boldsymbol{\Sigma} \boldsymbol{s}_{1}(\delta(\boldsymbol{s}, 0) E(\boldsymbol{m}-\boldsymbol{s}, \omega) \\
&+\delta(-\boldsymbol{s}, 0) E(\boldsymbol{m}+\boldsymbol{s}, \omega))=0 \\
& \boldsymbol{s}_{1}(-\mathbf{I}+\boldsymbol{\Sigma}) \boldsymbol{s}_{1} E(\boldsymbol{m}+\boldsymbol{s}, \omega) \\
&+\boldsymbol{s}_{1} \boldsymbol{\Sigma}\left(\boldsymbol{s}_{1} \delta(-\boldsymbol{s}, 0) E(\boldsymbol{m}+2 \boldsymbol{s}, \omega)\right. \\
&+\delta(\boldsymbol{s}, 0) E(\boldsymbol{m}, \omega))=0 \\
& \boldsymbol{s}_{1}(-\mathbf{I}+\boldsymbol{\Sigma}) \boldsymbol{s}_{1} E(\boldsymbol{m}-\boldsymbol{s}, \omega) \\
&+\boldsymbol{s}_{1} \boldsymbol{\Sigma}\left(\boldsymbol{s}_{1} \delta(\boldsymbol{s}, 0) E(\boldsymbol{m}-2 \boldsymbol{s}, \omega)\right. \\
&+\delta(-\boldsymbol{s}, 0) \boldsymbol{E}(\boldsymbol{m}, \omega))=0
\end{aligned}
$$

and for integer $p$ satisfying $|p|>1$

$$
\begin{aligned}
\boldsymbol{s}_{1}(-\mathbf{I} & +\boldsymbol{\Sigma}) \boldsymbol{s}_{1} E(\boldsymbol{m}+p \boldsymbol{s}, \omega) \\
& +\boldsymbol{s}_{1} \boldsymbol{\Sigma} \boldsymbol{s}_{1}(\delta(-\boldsymbol{s}, 0) E(\boldsymbol{m}+(p+1) \boldsymbol{s}, \omega)) \\
& +\delta(\boldsymbol{s}, 0) E(\boldsymbol{m}+(p-1) \boldsymbol{s}, \omega)=0
\end{aligned}
$$

Eq. (17) above is derived from Eqs. (3) and (4) and is essentially the modified field equation for the electromagnetic wave. Equations (18) and (19) are derived from Eqs. (3) and (13) for electrostatic waves with sideband currents involving those caused directly by the electromagnetic wave. Equation (20) is derived from Eqs. (3) and (13) and really stands for an infinite set of equations which apply to all the higher order purely electrostatic interactions with integer $p$ satisfying $|p|>1$. The solution to the set of coupled Eqs. (17) to (20) is found below.

\subsection{The coupling function}

Although it might at first sight be thought that the solution of the infinite set of coupled equations above presents formidable problems, the special symmetric form of the infinite set of equations represented by Eq. (20), with $|p|>1$, lends itself to a rather simple analysis. A little care needs to be exercised by treating the sidebands with positive and negative $p$ slightly differently. Although for positive $p, p+1$ is of a higher order than $p$, for the negative values of $p, p-1$ is of a higher order than $p$.

First, Eq. (20) is divided throughout by $E(\boldsymbol{m}+p \boldsymbol{s}, \omega)$. Then, after evaluating the matrices, the following expression is obtained for positive $p$,

$1-x_{-s} r_{p+1}-x_{+s} r_{p}^{-1}=0$

where

$$
\begin{aligned}
& r_{p}=\frac{E(\boldsymbol{m}+(p+1) \boldsymbol{s}, \omega)}{E(\boldsymbol{m}+p \boldsymbol{s}, \omega)} \\
& x_{ \pm s}=\frac{\delta( \pm \boldsymbol{s}, 0) W}{1-W} \\
& W=\frac{X(1+i Z)}{(1+i Z)^{2}-Y^{2}}
\end{aligned}
$$

In evaluating the matrices, it is assumed that

$\boldsymbol{s}_{1}=(\cos \alpha, \sin \alpha, 0)$

where $\alpha$ is the azimuthal angle of $s$ in the plane orthogonal to the background magnetic field (i.e. in the $x-y$ plane). Equation (21) can then be solved iteratively to yield a continued fraction of the form

$$
r_{p}=\frac{x_{+s}}{1-\frac{x_{-s} x_{+s}}{1-\frac{x_{-s} x_{+s}}{1-\cdots}}}
$$

It should be noted that the continued fraction in Eq. (26) has a self-similarity and simply yields

$r_{p}=\frac{x_{+s}}{1-x_{-s} r_{p}}$

Comparing this with Eq. (21) indicates that $r_{p}$ and $r_{p+1}$ are identical. Thus, $r_{p}$ is independent of $p$ and the subscript may be dropped. Equation (27) is then a quadratic in $r$ and yields the usual pair of complementary solutions. The negative square root is chosen here as this ensures that the coupling function, $r$, tends towards zero, away from the upperhybrid resonance region, as would be expected on physical grounds. Thus,

$r=\frac{1-W-\sqrt{(1-W)^{2}-W^{2} \delta_{0}^{2}}}{2 W \delta(-s), 0}$

where

$\delta_{0}^{2}=4 \delta(s, 0) \delta(-s, 0)$

Considering definition (29), it is also convenient to choose $\delta(-\boldsymbol{s}, 0)$ to be the complex conjugate of $\delta(\boldsymbol{s}, 0)$. Then, $\delta_{0}$ is the total amplitude of the fractional electron density perturbation in the striations. This also means that $\delta(s, 0)+\delta(-s, 0)$ is real and is just equal to $\delta_{0}$. A similar procedure is used for the negative values of $p$, when $p<-1$. Writing $q=E(\boldsymbol{m}+(p-1) s, \omega) / E(\boldsymbol{m}+p \boldsymbol{s}, \omega)$, subject to the latter inequality, then $q$ satisfies

$q=\frac{x_{-s}}{1-x_{+s} q}$

Thus, $q^{-1}$ satisfies the same quadratic as $r$ and consequently, $q$ is either identical to $r^{-1}$ or $q^{-1}$ and $r$ form a pair of complementary solutions to the quadratic. The latter option is chosen here, for reasons indicated below. If $q^{-1}$ is complementary to $r$, then $q$ is given by

$\frac{1}{q}=\frac{1-W+\sqrt{(1-W)^{2}-W^{2} \delta_{0}^{2}}}{2 W \delta(-s, 0)}$

After some manipulation, this leads to

$q=\frac{1-W-\sqrt{(1-W)^{2}-W^{2} \delta_{0}^{2}}}{2 W \delta(s, 0)}$ 
The negative square root now appears in Eq. (32) for $q$, which is virtually identical to Eq. (28) for $r$. The reason for the choice of a positive square root in Eq. (31) is now clear. As with $r$, it ensures that $q$ also goes to zero, away from the upper-hybrid resonance. Furthermore, only the products $r \delta(-s, 0)$ and $q \delta(s, 0)$ appear in the coupled field equations. Then, Eqs. (28) and (32) imply that negative and positive order contribute equally to the coupling. Writing

$F=r \delta(-s, 0)=\frac{1-W-\sqrt{(1-W)^{2}-W^{2} \delta_{0}^{2}}}{2 W}$

and inserting $F$ into Eqs. (17), (18) and (19) yields the modified dispersion relation for the electromagnetic waves in the presence of the striations.

\subsection{The modified electromagnetic dispersion function}

Inserting $F$ into Eqs. (18) and (19) yields expressions for $E(\boldsymbol{m}+\boldsymbol{s}, \omega)$ and $E(\boldsymbol{m}-\boldsymbol{s}, \omega)$ in terms of $\boldsymbol{E}(\boldsymbol{m}, \omega)$. Inserting these results into Eq. (17) then yields the purely electromagnetic field equation of the form

$(\boldsymbol{\Pi}+\boldsymbol{\Sigma}+\boldsymbol{\Delta}) \boldsymbol{E}(\boldsymbol{m}, \omega)=0$

where

$\Delta=-\frac{\delta_{0}^{2}}{2} \frac{\left(\Sigma s_{1}\right)\left(s_{1} \Sigma\right)}{s_{1}(-\mathbf{I}+\Sigma) s_{1}+F s_{1} \Sigma s_{1}}$

In explicit matrix form, Eq. (34) is then

$\left(\begin{array}{ccc}n_{m}^{2} \cos ^{2} \theta-S_{1} & -i D_{1} & -n_{m}^{2} \cos \theta \sin \theta \\ -i D_{2} & n_{m}^{2}-S_{2} & 0 \\ -n_{m}^{2} \cos \theta \sin \theta & 0 & n_{m}^{2} \sin ^{2} \theta-P\end{array}\right)\left(\begin{array}{c}E_{m x} \\ E_{m y} \\ E_{m z}\end{array}\right)=0$

where

$n_{m}^{2}=\frac{c^{2}}{\omega} \boldsymbol{m}^{2}$

and a unit vector, $\boldsymbol{m}_{1}$, in the direction of $\boldsymbol{m}$ is

$\boldsymbol{m}_{1}=(\sin \theta, 0, \cos \theta)$

Also,

$$
\begin{aligned}
& S_{1}=S-\Delta_{1 x} \quad S_{2}=S-\Delta_{2 y} \\
& D_{1}=D+i \Delta_{1 y} \quad D_{2}=D-i \Delta_{2 x} \\
& \Delta_{1 x}=G\left[(1+i Z)^{2} \cos ^{2} \alpha+Y^{2} \sin ^{2} \alpha\right] \\
& \Delta_{1 y}=G\left(\left[(1+i Z)^{2}-Y^{2}\right] \sin \alpha \cos \alpha-i(1+i Z) Y\right) \\
& \Delta_{2 x}=G\left(\left[(1+i Z)^{2}-Y^{2}\right] \sin \alpha \cos \alpha+i(1+i Z) Y\right) \\
& \Delta_{2 y}=G\left[(1+i Z)^{2} \sin ^{2} \alpha+Y^{2} \cos ^{2} \alpha\right] \\
& G=-\frac{\delta_{00}^{2} V}{2\left[(1+i Z)^{2}-Y^{2}\right]^{2}} \\
& \delta_{00}=X \delta_{0} \\
& V=\frac{1}{W(1+F)-1}
\end{aligned}
$$

It should be noted that $\delta_{00}$ is defined so that $|N(s, 0)|$ does not itself vary with altitude, even though $N_{0}$ is assumed to be weakly inhomogeneous. This is a somewhat arbitrary definition and reduces simply to a matter of choosing which height to fix a value of $N_{0}$. Here, the expression for $\delta_{00}$ is consistent with choosing the reflection height at which $X=1$. This follows previous calculations in Jones et al. (1984) and Robinson (1989). The modified refractive index is then obtained explicitly by setting the determinant of the $3 \times 3$ matrix in Eq. (35) to zero, which yields

$n_{m}=\sqrt{\frac{B_{1} \mp \sqrt{\left(B_{1}^{2}-4 A_{1} C_{1}\right)}}{2 A_{1}}}$

where

$$
\begin{aligned}
& A_{1}=S_{1} \sin ^{2} \theta+P \cos ^{2} \theta \\
& B_{1}=\left(S_{1} S_{2}-D_{1} D_{2}\right) \sin ^{2} \theta+P\left(S_{2} \cos ^{2} \theta+S_{1}\right) \\
& C_{1}=P\left(S_{1} S_{2}-D_{1} D_{2}\right)
\end{aligned}
$$

Before looking in more detail at the form of the refractive index in Eq. (36), it is worthwhile examining the form of the upper-hybrid resonance in this new formulation.

\subsection{The upper-hybrid resonance region}

The form of the upper-hybrid resonance in the present formulation, which takes into account higher order scatter, is significantly different from that obtained by previous authors who only included first order scatter (see Hagfors, 1984 and Mjølhus, 1985). The coupling function, $r$, in Eq. (28) governs the ratio of the size of any given wave amplitude to that of the next highest order sideband. Its structure across the upper-hybrid resonance is calculated below for the case when a linear background density as a function of altitude is assumed. The calculations below are greatly simplified if the propagation direction is taken along the density gradient, which is assumed to be vertical. Defining a scaled coordinate length, $h$, in units of $H$, in the direction of $\boldsymbol{m}_{1}$, then

$X=1+h$

Thus, actual distances in the vertical direction is just $h H$. Clearly, Eq. (37) implies $X=1$ at the origin, which thus coincides with the reflection point of the ordinary electromagnetic mode. Inserting Eq. (37) into Eq. (28) then yields, for $r$ as function of $\zeta=\left(h+Y^{2}\right) / \delta_{00}$

$r=\frac{-\zeta+i Z\left(1+Y^{2}\right)}{\delta_{00}}-\sqrt{\frac{-\zeta+i Z\left(1+Y^{2}\right)}{\delta_{00}}-1}$

The real and imaginary parts of $r$ as a function of $\zeta$ in the vicinity of the upper-hybrid layer (at $h=Y^{2}$ and $\zeta=0$, are displayed in Fig. 1. Typical values of $Z=10^{-5}$ and $Y=0.3$, which are applicable to F-region conditions and a high frequency radio wave have been used. In addition, since the experimentally determined amplitudes of the striations are quoted in terms of their root-mean-square (rms) values, 

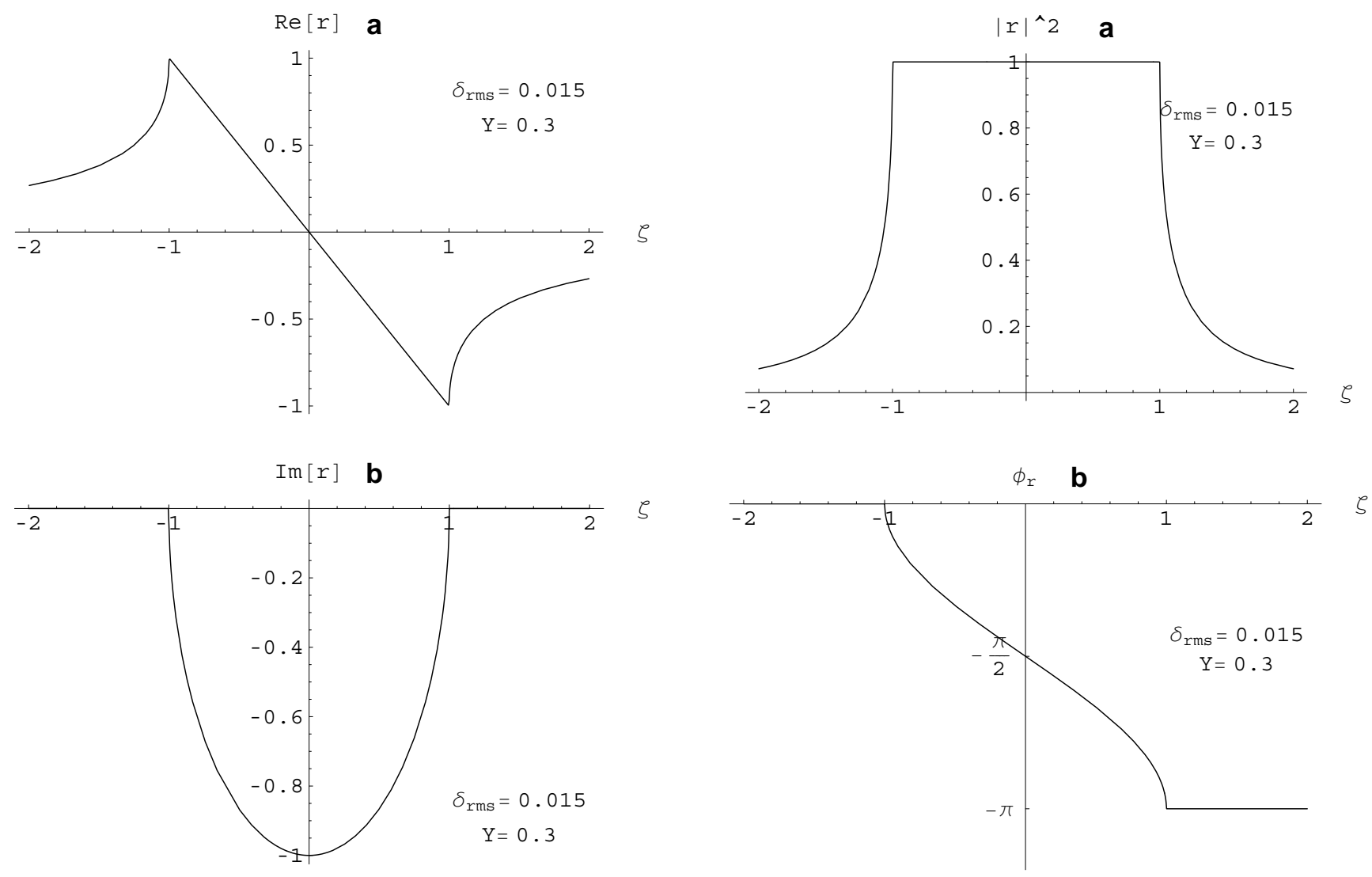

Fig. 1. The real (a) and imaginary (b) parts of the multiple scatter coupling function, $r$, as a function of coordinate, $\zeta$.

$\delta_{\text {rms }}=\delta_{00} / \sqrt{2}$ has been used to define this parameter in the calculations which follow. Here, a fairly typical value of $\delta_{\text {rms }}=0.015$ has been employed (Robinson, 1989). Thus, the condition $Z / \delta_{00} \ll 1$ is strongly satisfied.

The two most striking characteristics of the function $r$ in Fig. 1 are first, that its imaginary part is only significantly different from zero for values of $h$ between $-Y^{2} \pm \delta_{0}$ (i.e. between $-1 \leq \zeta \leq 1)$. This is a result of the expression within the square root in Eq. (38) being essentially negative in this interval, even in the absence of collisions. Thus, the resonance width is $2 \delta_{00} H$. The real part of $r$ is linear in this interval. Second, the sizes of both real and imaginary parts of $r$ peak at a value very close to unity. Indeed, this value is exactly unity in the limit of no collisions, when $Z \rightarrow 0$, as is clear from Eq. (38). The value of $|r|^{2}$ is almost constant and close to unity throughout the interval $h=-Y^{2} \pm \delta_{00}$, as indicated in Fig. 2, which also contains the phase of $r, \phi_{r}$. In addition, the value of $\phi_{r}$ changes by $\pi$ across the resonance interval. It is interesting to note that the results in Figs. 1 and 2 show that the scattered waves are all equal in amplitude and essentially (see Sect. 5 below) also equal to the amplitude of the test wave, within the resonance interval between $-Y^{2} \pm \delta_{00}$. The fact that the width of the upper-hybrid resonance is much broader than that predicted by single scatter theory can be seen by comparing the curves in Fig. 1 to those

Fig. 2. The amplitude squared (a) and phase (b) of the multiple scatter coupling function, $r$, as a function of coordinate, $\zeta$.

in Fig. 3, in which the values of the real and imaginary parts of the function $s$, where

$r_{s}=\frac{1}{2} \frac{\delta_{00} W}{1-W}=\frac{1}{2}\left(-\zeta+\frac{i Z\left(1+Y^{2}\right)}{\delta_{00}}\right)^{-1}$

have been plotted. The corresponding amplitude and phase curves for $r_{s}$ are also plotted in Fig. 4. $r_{s}$ is the ratio of the amplitude of the electric field of the pair of electrostatic wave sidebands to that of the electromagnetic test wave in the single scatter approximation and is found by setting the last term on the LHS of Eq. (21) to zero. In the present example, with $Z \ll \delta_{00}$, it is clear that the width of $r_{s}$ is much smaller than that of $r$. Further, $\left|r_{s}\right|$ peaks at values much larger than unity. Moreover, in the limit as $Z \rightarrow 0$, the peak of $\left|r_{s}\right|$ continues to grow as its width becomes smaller, in contrast to $r$, whose amplitude just converges to unity and whose width converges to $2 \delta_{00}$. Despite the obvious differences between the functions $r$ and $r_{s}$, it turns out that the areas under the curves representing their respective imaginary parts are equal in the limit of small $Y$. This result can easily be appreciated by noting that the amplitude of $\operatorname{Im}(r)$ is 1 , whilst its width (in terms of $h$ ) is $2 \delta_{00}$. Thus, its area is of the order of $\delta_{00}$. Now the width of the $\operatorname{Im} r_{s}$ curve is $2 Z$ and its amplitude is $\delta_{00} / Z$. Thus, its area is also approximately $\delta_{00}$. Since it is these imaginary parts that determine the levels of anomalous 

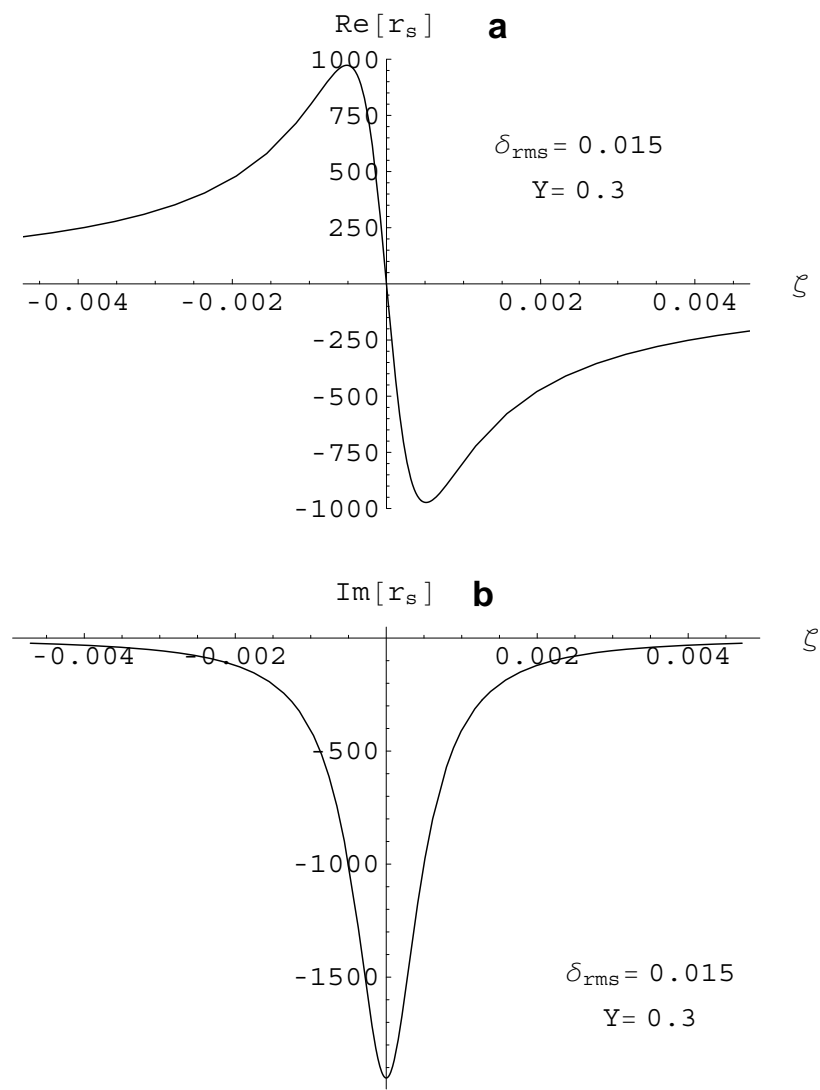

Fig. 3. The real (a) and imaginary (b) parts of the single scatter resonance function, $r_{s}$, as a function of coordinate, $\zeta$.

absorption of the test wave (see Sect. 4), this explains why single scatter theory (if changes to the real part of the refractive index are ignored) gives the same result for anomalous absorption as the more rigorous multiple scatter theory.

\section{Calculation of propagation and absorption effects}

Using Eq. (36), the real and imaginary parts of the modified refractive index of an O-mode electromagnetic test wave in a cold plasma with field-aligned plasma density striations have been calculated as functions of scaled altitude, $h$. In this calculation, the angle between the magnetic field and the test wave propagation direction, $\theta$, is taken as $12^{\circ}$, which is consistent with the geomagnetic field geometry at the site of the high power facility at Troms $\varnothing$. In addition, the value of the angle $\alpha$, between the normal to the plane of the striations and the plane containing both the magnetic field and the test wave propagation direction is chosen arbitrarily to be $0^{\circ}$ in this example. The resulting curves are displayed in Fig. 5. Values of $Y, Z$ and $\delta_{00}$ are the same as those for Figs. 1 to 4, above. Panel (a) in Fig. 5 also contains the real part of the refractive index in the absence of striations, as a dashed curve. The fact that this dashed curve is virtually obliterated by the solid curve which represents the case with striations is testament to the fact that the striations have a negligible effect on the

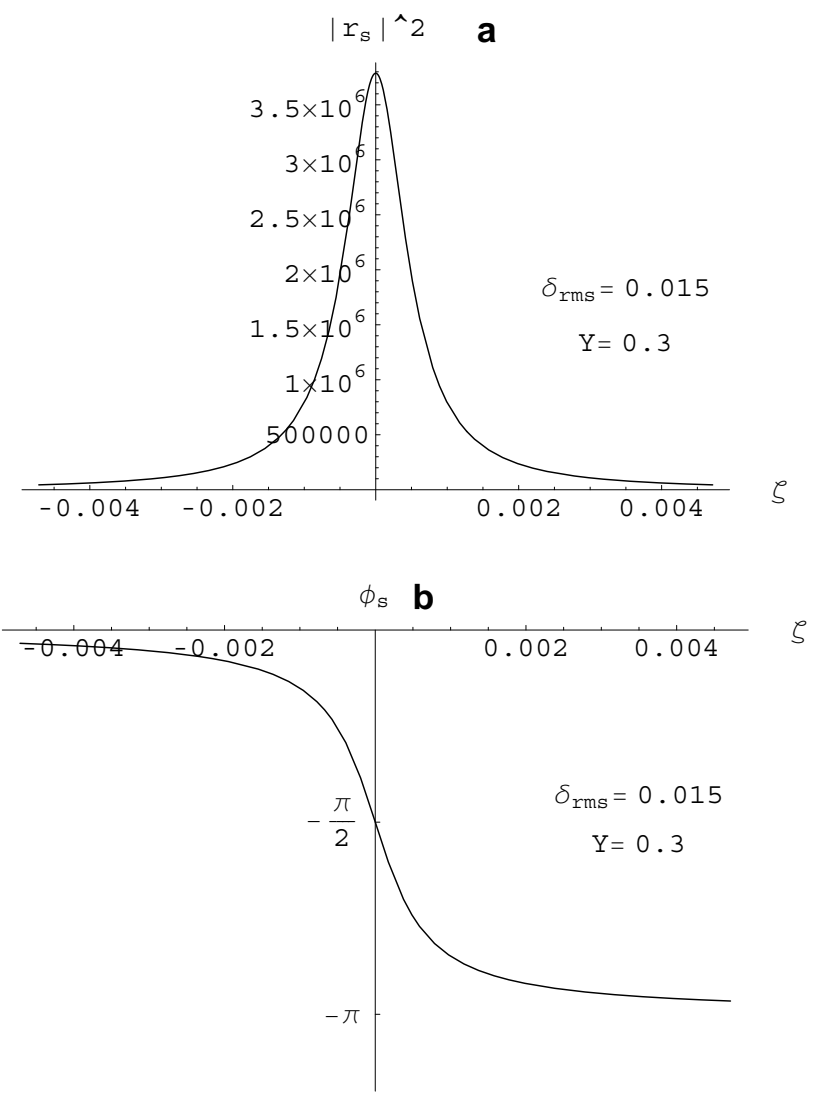

Fig. 4. The amplitude squared (a) and phase (b) of the single scatter resonance function, $r_{s}$, as a function of coordinate, $\zeta$.

real part. Thus, the striations do not affect the propagation (i.e. phase and group speed) of the test wave in the vicinity of the upper-hybrid resonance, which is near $h=0.09$ in the present example. With the present striation amplitude, it is clear that a new cutoff at the upper-hybrid resonance, predicted by the single scatter theory (Hagfors, 1984), does not actually occur. The striations do, however, have a significant effect on the imaginary part of the refractive index, as is clear from panel (b). Although much less than $\operatorname{Re}\left(n_{m}\right), \operatorname{Im}\left(n_{m}\right)$ acquires a positive value in the vicinity of the upper-hybrid resonance, which is much greater than that due to collisions. This indicates strong absorption. The shape of the $\operatorname{Im}\left(n_{m}\right)$ curve in the interval $h=-Y^{2} \pm \delta_{00}$ is similar in form to that of $\operatorname{Im}(r)$ in Fig. 1, panel(b), though of opposite sign.

The dependence of the above results on the orientation of the striations, as defined by the angel $\alpha$, in relation (25) was also investigated. It was found that the results were only very weakly dependent on this parameter. This is not too surprising since at the upper-hybrid resonance, the electric field of the electromagnetic test wave is almost exactly circularly polarised in a plane orthogonal to the background magnetic field and is thus rather insensitive to azimuth in this plane.

The value of the anomalous absorption, $\Gamma$, for the test wave propagating vertically and at a small angle to the background magnetic field, in traversing the upper-hybrid reso- 

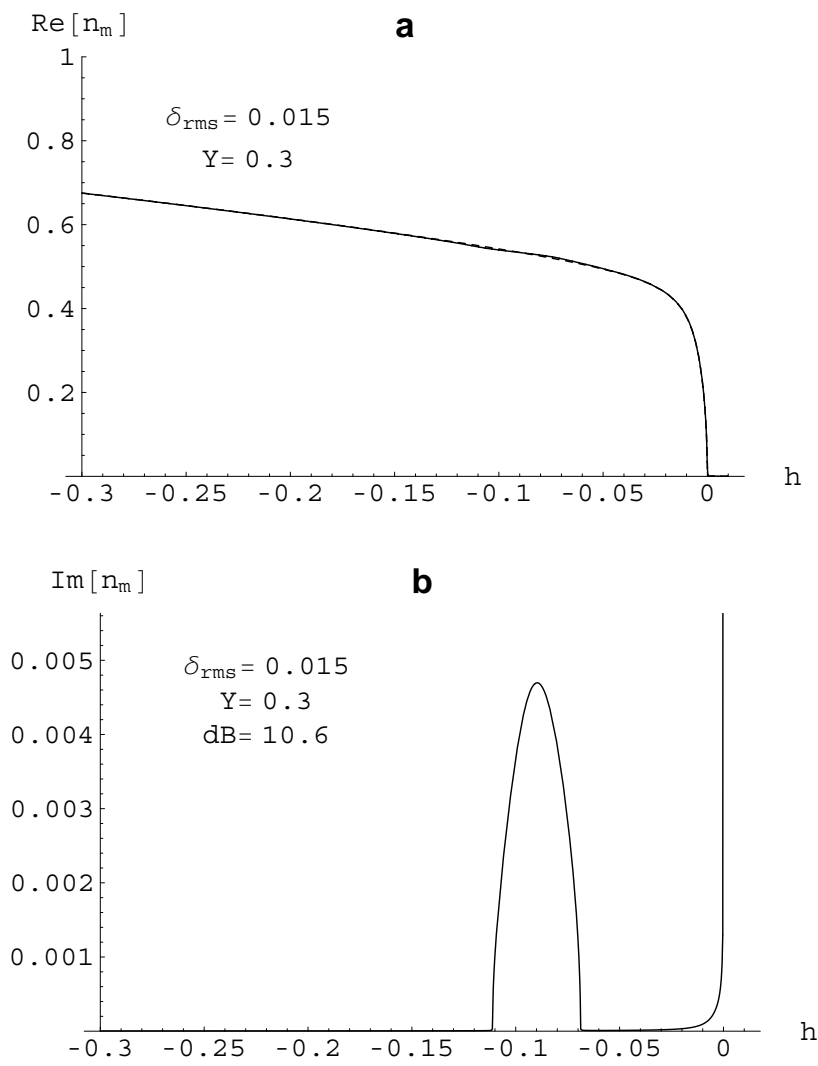

Fig. 5. The real (a) and imaginary (b) parts of the refractive index, $n_{m}$, of an O-mode test wave, as a function of scaled height coordinate, $h$, for the rms fractional striation amplitude, $\delta_{r m s}=0.015$.

nance, is easily obtained from

$\Gamma=4 \frac{H \omega}{c} \int_{-\infty}^{\infty} \operatorname{Im}\left(n_{m}\right) \mathrm{d} h$

The factor of 4 in Eq. (40) allows for the fact that the power in the wave is proportional to its amplitude squared and that the wave traverses the upper-hybrid resonance twice, in propagating from the ground and reflecting from the layer where $X=1$. Equation (40) correctly predicts the levels of absorption since the width of the resonance is much larger than the wavelength of a typical test wave. In the example under consideration, with $H=40 \mathrm{~km}$, the width of the resonance is $1.6 \mathrm{~km}$ and the wavelength of the test wave at the upper-hybrid resonance, allowing for refraction, is approximately $120 \mathrm{~m}$. Thus, the resonance is many wavelengths wide. However, the observed scale length of the striations along the geomagnetic field of a few tens of $\mathrm{km}$ (Jones et al. 1984) means that the amplitude of the striations, $\delta_{00}$, can still be considered as constant over the resonance interval. The corresponding value of $\Gamma$ is $10.6 \mathrm{~dB}$, which is consistent with that observed during ionospheric modification experiments using the high power facility at Troms $\emptyset$ (Robinson, 1989).
In the limit of small $Y$, Eq. (40) approximates to

$\Gamma=\pi \delta_{00}^{2} \frac{H \omega}{c} \frac{1}{\sqrt{Y}}$

which is identical to that obtained by Gurevich (1978) and Jones et al. (1984), using energy considerations and single scatter theory. Expression (41) indicates that $\Gamma$ is proportional to $\delta_{00}$ and is independent of $v_{e n}$.

Finally, in this section, it is worth briefly examining whether there are circumstances under which the propagation of a test wave could be affected if the striation amplitude were large enough. Curves for the case when $\delta_{\text {rms }}=0.1$ are displayed in Fig. 6. The first thing to notice about these curves is that the width of the resonance region is significantly broader than that in Fig. 5, as expected. However, although the distortion to the $\operatorname{Re}\left(n_{m}\right)$ curve, which is brought about by the striations, is more noticeable than in Fig. 5, it is still not substantial and certainly is far from introducing any new cutoff. What is significant here is the anomalous absorption level, which turns out to be $2230 \mathrm{~dB}$. This is of course unrealistically large. It should be kept in mind that any high power pump wave which creates striations in an ionospheric modification experiment also undergoes anomalous absorption at similar and usually greater levels than lower power test waves. Since the high power wave has to overcome its own self-absorption in these circumstances (Robinson, 1989), this exceptionally high level of anomalous absorption means that the high power wave would extinguish itself before it penetrated even a tiny fraction of the resonance region. Thus, values of $\delta_{\text {rms }}$ of the order of 0.1 are probably not practically achievable. Thus, it is unlikely that a new cutoff at the upper-hybrid resonance could ever be produced by the action of a high power radio wave in the ionosphere, at least with the present capabilities.

\section{Nature of the resonance broadening process}

\subsection{The case of sinusoidal striations}

At first sight, the broadening of the upper-hybrid resonance seems puzzling. After all, all of the individual resonances associated with each order of the scattered electrostatic wave fields appear to occur at precisely the same altitude, i. e. where condition (15) is satisfied. However, further considerations indicate that there is actually some uncertainty in the position of the resonance, due to the variation in the plasma density in the plane perpendicular to the magnetic field, which is due to the striations. However, it is not immediately obvious how variations in plasma density in the perpendicular plane might affect the position of the resonance along the $\zeta$ coordinate. Although $x$ and $\zeta$ are not exactly orthogonal, in general, they may be taken to be close to orthogonal in most cases of interest. To explain this remarkable broadening effect, it is necessary to evaluate the plasma density 'seen' by the electric field in the resonance region. Since the results above are essentially independent of the azimuthal 

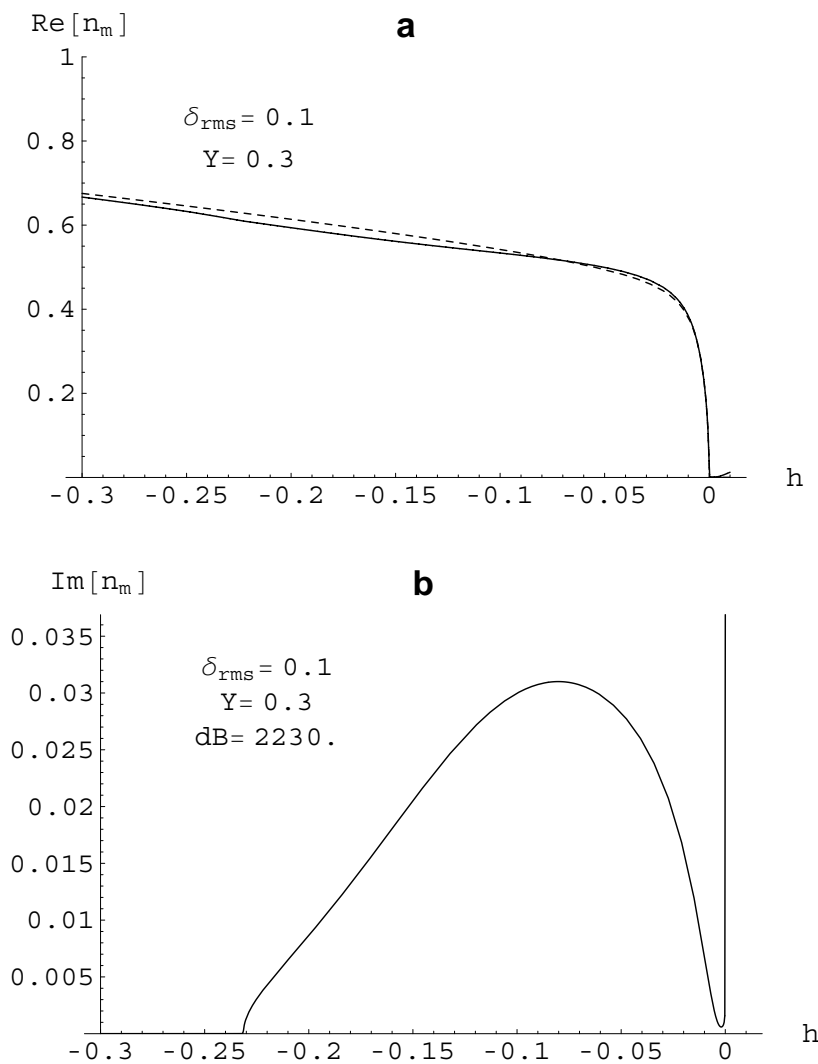

Fig. 6. Same as in Fig. 5, but with $\delta_{r m s}=0.1$.

angle, $\alpha$, of vector $s_{1}$, in what follows, $s_{1}$ will be taken along the $x$-axis. Then, with the aid of Eqs. (18) and (28), the first order electrostatic field can be expressed in terms of the electromagnetic wave amplitude as

$E(\boldsymbol{m}+\boldsymbol{s}, \omega)=r E_{0}$

where $E_{0}$ is $E_{m x}-i Y E_{m y}$. The total electric field, $\Sigma_{E}$, of all of the superposed electrostatic waves is then just

$\Sigma_{E}=E_{0} \sum_{p=0}^{\infty} r^{p}(\exp (i p s x)+\exp (-i p s x))$

where $s$ is $|s|$. The sums of the pair of infinite geometric series in Eq. (43) are easily evaluated, so that the size of the field $\left|\Sigma_{E}\right|$ is just

$\left|\Sigma_{E \mid}=\right| E_{0} \mid \sqrt{\frac{A_{2}}{B_{2}}}$

with

$$
\begin{array}{r}
A_{2}=1-(r+r *) \cos (s x)+r^{*} r \cos ^{2}(s x) \\
B_{2}=1+4 r^{*} r \cos ^{2}(s x)+\left(r^{*} r\right)^{2}+\left(r^{* 2}+r^{2}\right) \\
-2\left(r+r^{*}\right)\left(1+r^{*} r\right) \cos (s x)
\end{array}
$$

where $r^{*}$ is the complex conjugate of $r$. It has been assumed, in writing Eq. (44), that the ratio of each successively higher order term to its predecessor is less than unity, as will become clear below. The most straightforward way to simplify Eq. (44) is to write Eq. (38) as

$r-2 R+r^{-1}=0$

where

$$
R=-\zeta+\frac{i Z\left(1+Y^{2}\right)}{\delta_{00}}
$$

Then, from Eq. (45), it may be shown that

$$
1+\left(r^{*^{2}}+r^{2}\right)+\left(r r^{*}\right)^{2}=\left(r+r^{*}\right)^{2}+\left(1-r^{*} r\right)^{2}
$$

and

$\left(1-r^{*} r\right)=-2 \frac{\operatorname{Im} R}{\operatorname{Im} r} r r^{*}$

Then, with $Z / \delta_{00} \ll 1$, the following relations are satified to high accuracy, within the resonance interval

$r^{*} r=1$

$\operatorname{Im} r=-\sqrt{1-\zeta^{2}}$
$\operatorname{Re} r=-\zeta$

The RHS of Eq. (47) is very small and positive. Although Eq. (48) is correct to a very good approximation, Eqs. (47) to (50) together imply that $r^{*} r$ is just slightly smaller than 1, allowing the sum to infinity in Eq. (43) to be performed legitimately. Substituting Eqs. (46) to (50) into Eq. (44), then yields, after some manipulation,

$\left|\Sigma_{E}\right|=\left|E_{0}\right|\left(\frac{1+2 \zeta \cos (s x)+\cos ^{2}(s x)}{(\cos (s x)+\zeta)^{2}+\frac{Z^{2}\left(1+Y^{2}\right)^{2}}{\delta_{00}^{2}\left(1-\zeta^{2}\right)}}\right)^{1 / 2}$

Thus, the peak values of $\left|\Sigma_{E}\right|$ lie along a contour which satisfies

$\cos (s x)=-\zeta$

Furthermore, the peak amplitude itself, $\left|\Sigma_{E}\right|_{\max }$, along this contour is then

$\left|\Sigma_{E}\right|_{\max }=\left|E_{0}\right| \delta_{00} \frac{1-\zeta^{2}}{Z\left(1+Y^{2}\right)}$

and the half-width, in terms of $s x$, is approximately

$\Delta(s x)=\frac{Z\left(1+Y^{2}\right)}{\delta_{00} \sqrt{1-\zeta^{2}}}$

Equations (53) and (54) indicate that the peak in $\Sigma_{E}$ diminishes in amplitude and broadens in width (in the $x$-direction) towards the boundaries of the resonance region.

The profound implications of Eq. (52) become clear when it is noted that the local plasma frequency, when the effect 
of the striations is taken into account, may be represented by $X^{\prime}$, the perturbed value of $X$, as

$X^{\prime}=X\left(1+\delta_{0} \cos (s x)\right)$

Inserting Eq. (52) into Eq. (55) yields, after some manipulation

$X^{\prime}=1-Y^{2}$

Thus, the peak in the total electric field lies precisely along the local upper-hybrid contour which makes clear how the upper-hybrid layer is spread. Due to the incident electromagnetic wave, which is itself highly uniform in the plane perpendicular to the geomagnetic field, electrostatic fluctuations in any layer where it encounters the local upper-hybrid resonance condition start to excite. This occurs throughout the region where $-1 \leq \zeta \leq 1$.

The total electric field, whose amplitude is given by Eq. (51) is illustrated quasi-schematically in Fig. 7, together with the upper-hybrid contour. Apart from points close to where $|\zeta|=1$, the upper-hybrid contour lies almost parallel to the geomagnetic field and the angle between them is roughly $\pi /\left(2 s \delta_{00} H\right)$. This angle is only around $10^{-3}$ radians in the present example.

The reason why the single scatter approach produces a narrow resonance interval along the direction of the striations may be also seen in similar terms. The size of the total electric field $\left|E_{S}\right|$ is then just

$\left|E_{s}\right|=\left|E_{0}\right| \frac{\delta_{00} \cos (s x)}{\sqrt{\zeta^{2} \delta_{00}^{2}+Z^{2}\left(1+Y^{2}\right)^{2}}}$

Clearly, in contrast to Eq. (51), the electrostatic wave field in Eq. (57) is both sinusoidal in nature and also its phase along the $\mathrm{x}$-axis is not dependent on the $\zeta$ coordinate. Thus, the mean plasma density 'seen' by the field, in this case, is always $N_{0}$, and there is no spreading of the resonance interval in the $\zeta$ direction, due to changes in plasma density in the striations.

\subsection{The case of broad-band striations}

With the insight gained from the sinusoidal case above, the discussion concerning the resonance broadening is readily extended, albeit in an approximate way to the case of a broadband spectrum of plasma density striations. The spectrum of irregularities may then be taken to be centred on, for example, wavenumber, $s$, and with a non-zero spectral width, $\sigma_{s}$. This implies that the autocorrelation function of the density of the striations is a quasi-gaussian function in $x$, but, as before, independent of the direction parallel to the magnetic field. The width of the $k$-spectrum of the density irregularities, although no longer a single Fourier component, is still much narrower than the final spectrum of electric field components, as in the case of sinusoidal irregularities. This may be understood in the following way. With the same test wave as before, the spectrum of the first order scattered electric field will occupy the same spectral band as that of the irregularities. The second order scatter, centred on $2 s$, will be broader, with a width of approximately $(\sqrt{2}) \sigma_{s}$. Subsequent scattering will broaden the widths of successive orders of the scattered electric field, so that the $\mathrm{n}^{\text {th }}$ order scatter, centred on $n s$, has a width $\sqrt{n}$ times that of the first order. The effect will be to smear the spectrum of the higher order scattered wave field into a continuous flat distribution in wavenumber. The result of this smearing is illustrated schematically in Fig. 8. The first order scattered electrostatic field, which corresponds to low wavenumbers in the band occupied by the striations, is indicted by the subscript ' $L$ ' and the higher orders of the scattered electrostatic field, which has wavenumbers beyond the striation waveband, are indicated by the subscript ' $H$ '. The broad-band coupling may then be approximated by an equation coupling each first order electrostatic sideband to the electromagnetic wave and a band of higher order scatter, together with a second equation which handles the coupling between the higher order modes. These coupling equations are, respectively, with the aid of Eqs. (6) and (24)

$$
\begin{aligned}
& \left(1-\frac{1}{W}\right) E_{L}(k, \omega)+\delta(k, 0) E_{0} \\
& +\int_{0}^{\infty} \delta(-l, 0) E_{H}(k+l, \omega) \mathrm{d} l=0 \\
& \left(1-\frac{1}{W}\right) E_{H}(k, \omega) \\
& +\int_{-\infty}^{\infty} \delta(-l, 0) E_{H}(k+l, \omega) \mathrm{d} l=0
\end{aligned}
$$

where $k$ and $l$ represent variable wavenumbers. It should be noted that both irregularity sidebands are combined in one integral in Eq. (59), but have to be separated in Eq. (58). Furthermore, the broad-band case requires $r$ to become a function $r(k)$ of the wavenumber of the striation spectrum and $F$ in the first line of Eq. (33) to be replaced by an integral over the striation spectrum. Hence,

$F=\int_{0}^{\infty} r(k) \delta(-k, 0) \mathrm{d} k$

where $\delta(k, 0)$ now represents a spectral density. The broadband replacement for Eq. (20) implies that $E_{H}(k+l, \omega)$ may be replaced by $r(l) E_{L}(k, \omega)$ in Eq. (58), which then yields, with the aid of Eq. (60)

$E_{L}(k, \omega)=-\frac{\delta(k, 0) E_{0}}{1-\frac{1}{W}+F}$

Multiplying Eq. $(61)$ by $\delta(-k, 0)$ and integrating over $k$ then yields, with the aid of Eqs. (42) and (60), a quadratic equa- 
a

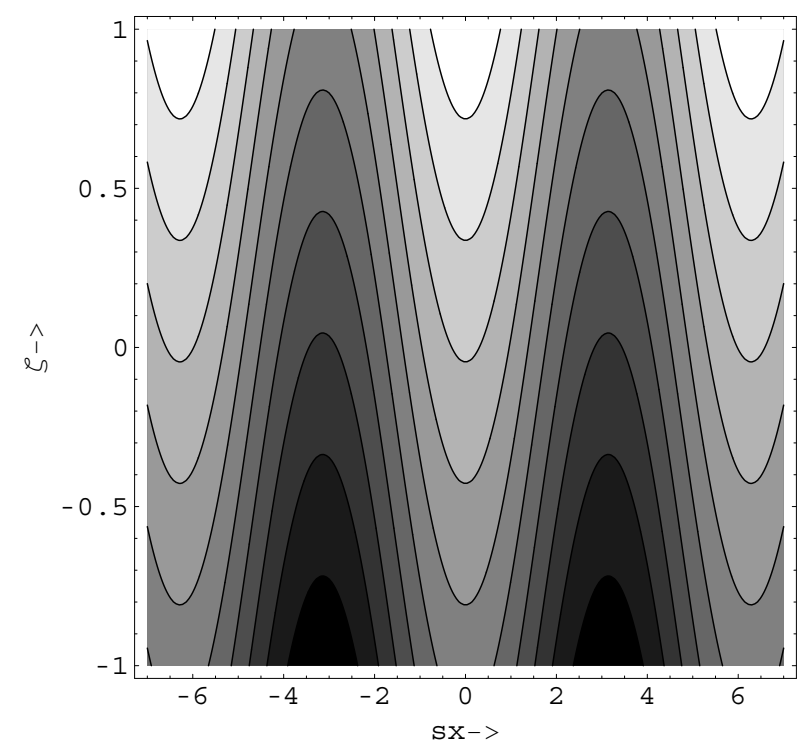

b

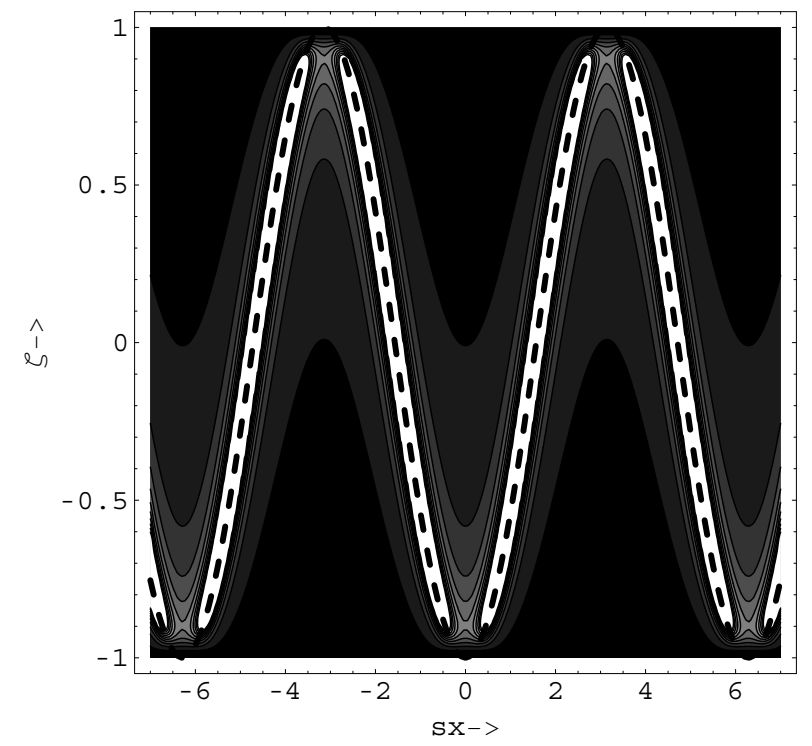

Fig. 7. (a) Depicts the contours of the plasma density perturbations in the vicinity of the UHR in the $x-\zeta$ plane (lighter shading indicates higher values). (b) Illustrates the corresponding contour plot of the total electric field, together with the UHR contour (dashed).

tion for $F$ of the form

$F=\frac{1}{1-\frac{1}{W}+F} \int_{0}^{\infty} \delta(-k, 0) \delta(k, 0) \mathrm{d} k$

Thus, $F$ satisfies the same expression as in the sinusoidal case (Eq. 33) as long as $\delta_{0}^{2}$ is replaced by the integrated spec-

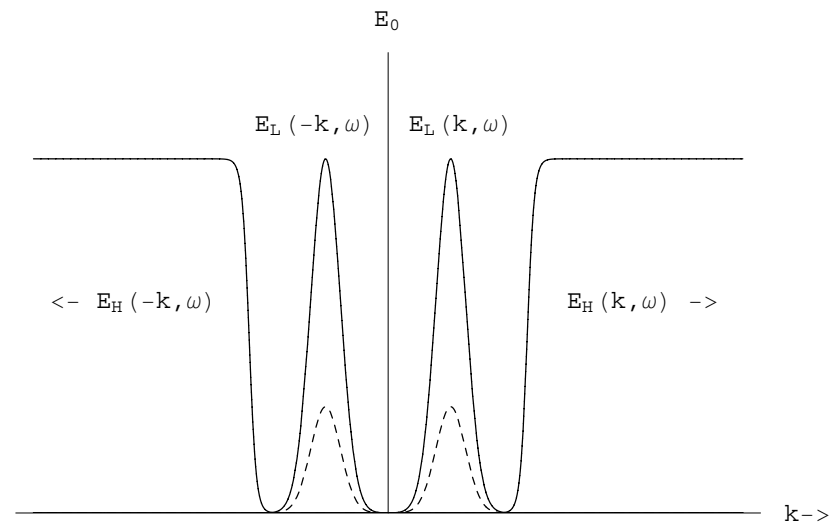

Fig. 8. A schematic representation of the scattered electric field spectrum in the broad-band case. The continuous curve represents the electric field and the dashed curve represents the striation spectrum. Subscript ' $L$ ' indicates broad-band first order wavenumbers and ' $H$ ' indicates all higher orders.

tral power

$$
\begin{aligned}
\left\langle|\delta|^{2}\right\rangle & =\int_{0}^{\infty}(\delta(k, 0)+\delta(-k, 0))^{2} \mathrm{~d} k \\
& =4 \int_{0}^{\infty} \delta(k, 0) \delta(-k, 0) \mathrm{d} k
\end{aligned}
$$

Thus, the width of the resonance in the broad-band case is effectively determined by the rms fractional plasma density deviation level, as could be reasonably expected.

Finally, the location of the peak in the total electric field may be estimated for the broad-band case, as follows. The coupling of Eq. (59) for the higher order scatter is inverse Fourier transformed to yield

$$
\left(1-\frac{1}{W}+\delta_{N}(x)\right) E_{H}(x)=0
$$

where $\delta_{N}(x)$ and $E_{H}(x)$ are the fractional perturbation in electron density and the total higher electric field in spatial coordinates, respectively. This implies that the high order scattered field, which contains the overwhelming proportion of the power of the total scattered electric field, is only nonzero where the first factor in Eq. (63) is exactly zero. This is again, as with the sinusoidal case, precisely along the local upper-hybrid contour (Eq. 56).

\section{Thermal effects}

Warm plasma effects can modify the above results in a number of ways. First, radio wave heating of the plasma by a high power electromagnetic pump wave which sets up the striations can increase the electron temperature and enhance some of the transport coefficients. In particular, the electronneutral collision frequency can be increased considerably by 
heating in the upper-hybrid layer (Gurevich, 1978). However, even if the electron temperature were to increase tenfold in this region, this would only increase the resonance width to around $10 \mathrm{~m}$, which is still much smaller than the wavelength of the test wave. Second, in a warm plasma, high frequency electrostatic waves propagate at the electron thermal speed and the wave dispersion effects arise. This has the effect of shifting the resonance slightly away from the upperhybrid frequency. Then Eq. (15) becomes, approximately, for an electrostatic wave of wavevector, $\boldsymbol{k}$

$X=\frac{1+i Z-Y^{2}}{1+\boldsymbol{k}^{2} \lambda_{D}^{2}}$

where $\lambda_{D}$ is the Debye length. Now $\lambda_{D}$ is of the order of a few millimetres in the ionospheric F-region. Consequently, with $2 \pi /|\boldsymbol{k}|$ of the order of $1 \mathrm{~m}$ or greater for a typical spectrum of striations encountered in ionospheric modification experiments (Minkoff, 1974), the spread from the upperhybrid resonance altitude caused by the warm plasma effect will only be of the order of a few metres in the single scatter case.

The multiple scatter case, in a warm plasma, has previously been investigated by Inhester et al. (1981) in the context of the striation generation process. Inhester et al. (1981) showed that in the warm plasma case, with sinusoidal striations, Eq. (27) for $r$ needs to be replaced by $r_{t}$, where

$$
r_{t}^{(p)}=\frac{x_{p s}}{1-\frac{x_{p s} x_{(p+1) s}}{1-\frac{x_{(p+1) s} x_{(p+2) s}}{1-\frac{x_{(p+2) s} x_{(p+3) s}}{1-\cdots}}}}
$$

and

$$
x_{(p+j) s}=\frac{\delta_{00}}{1-X\left(1+\lambda_{D}^{2}((p+j) s)^{2}-Y^{2}-i Z\right)}
$$

Now unlike Eq. (26), Eq. (65) does not have a self-similarity property. Furthermore, $r_{t}$ now depends on $p$ and it cannot be easily evaluated analytically. The real and imaginary parts of $r_{t}$ in Eq. (65), for the $p=1$ case, are plotted as solid curves in panels (a) and (b), respectively, in Fig. 9. These plots are somewhat schematic in order to illustrate the effect, rather than as an accurate representation. An arbitrary value of $\left(s \lambda_{D}\right)^{2} / \delta_{00}=0.003$ has been used in obtaining these curves. Also, following Inhester et al. (1981), a small $p$ dependent, imaginary term has been introduced into Eq. (66) to account for Landau damping. The dashed curves in each panel in Fig. 9 represent the real and imaginary parts of $r_{t}$, respectively. Although, as is clear from Fig. 9, $r_{t}$ is much more structured than $r$, it is remarkably confined to the same resonance interval, $|\zeta|<1$, as $r$. This result is independent of the value of $s \lambda_{D}$ chosen, apart from the number of peaks in the resonance interval, which appears to be approximately equal to $\sqrt{\delta_{00}} /\left(s \lambda_{D}\right)$. It is clear that it is the multiple scatter effects that control the broadening of the resonance and not
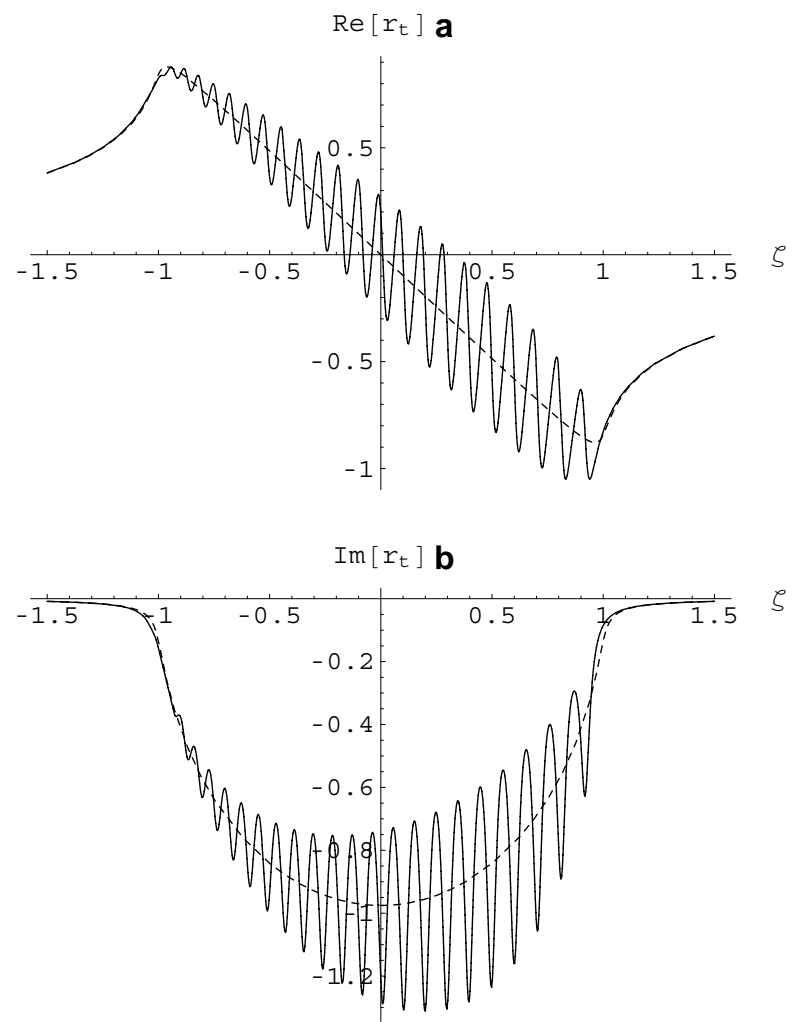

Fig. 9. Solid curves: The real (a) and imaginary (b) parts of the multiple scatter coupling function $r_{t}$, with thermal effects included, for the case where $p=1$ and $\left(s \lambda_{D}\right)^{2} / \delta_{00}=0.003$. The dashed curves are the corresponding cold plasma functions, as in Fig. 1.

the shift due to warm plasma dispersion effects. Moreover, the areas under the two curves in panel (b) of Fig. 9, which represent imaginary parts, were evaluated by numerical integration and were found to be identical, within the limits of accuracy of the calculation. This is an important result as it is the areas under these curves which determines the levels of anomalous absorption caused by the FASTUHR process in each case. This result shows that warm plasma effects play no major role in determining anomalous absorption under these conditions.

\section{Conclusions}

A theory in which the effects of multiple scatter on the propagation and absorption of electromagnetic test waves in a cold magnetoplasma containing field-aligned plasma density striations has been developed above. The theory has been used to calculate the complex refractive index of a test wave in conditions that are applicable to ionospheric modification experiments in which artificial striations, generated by the action of a high power electromagnetic pump, cause anomalous absorption of the test wave. The main findings of the new theory are as follows:

(1) The refractive index of an ordinary mode wave is modified in the vicinity of the upper-hybrid resonance as a re- 
sult of the presence of striations of sufficient amplitude, but the width of the resonance region, in the multiple scatter case, is much larger than obtained in previous, single scatter, calculations. This width is found to be typically several tens of times the wavelength of the test wave, under normal F-region conditions, rather than a small fraction of a wavelength, as previously predicted. Moreover, the multiple scatter resonance function remains finite in the limit of zero collision frequency, unlike the single scatter case. The reason for this behaviour is the localisation of the scattered electrostatic field at the upper-hybrid resonance layer which follows precisely the undulations imposed by the plasma density striations.

(2) With fractional striation amplitudes of a few percent, the imaginary part of the refractive index in the resonance layer is much larger than that due to electron-neutral collisions and this leads to strong anomalous absorption of the test wave. However, the real part of the refractive index is almost unaffected and, contrary to previous theoretical predictions, no new cutoffs appear at the upperhybrid layer. Thus, there are no new test wave reflection effects associated with ionospheric modification experiments.

(3) Multiple scatter effects also appear to be the dominant mechanism for the upper-hybrid resonance broadening, even when warm plasma effects are included. Furthermore, the results presented above also appear to be rather independent of the detailed spectrum of the striations, as long as they are strongly aligned with the background magnetic field.

The calculations above are also entirely consistent with test wave anomalous absorption observations made during numerous ionospheric modification experiments, at least for cases where the test wave frequency is not close to a harmonic of the electron gyro-frequency. Kinetic theory is needed to explain these latter, rather special conditions. Multiple scatter theory, thus, seems to clear up a number of rather inconsistent aspects of the previous, single scatter, theories of anomalous absorption and propagation of test waves.

Finally, the theory above may also have some important consequences for the action of the high power electromagnetic pump itself. Although a detailed investigation of this topic is beyond the scope of the present study, it is worth speculating briefly on how the new results might impact on this important issue. For example, the broadening of the resonance, which is such an important aspect of the new theory, may increase the sensitivity of the pump to self-absorption processes which may limit the growth of the instability by which the pump creates the striations. Furthermore, the way in which the multiple scatter leads to a total electric field of the superposed scattered waves that is made up essentially of broad-band noise, could have a profound effect on the nature of the heating processes itself. In the previous single scatter approach, it was assumed that the coherent electromagnetic pump wave had its energy converted into a quasi-coherent, though large amplitude, electrostatic wave in the vicinity of the upper-hybrid resonance and that the large electric field of this slowly propagating wave heated the plasma through electron-neutral collisions (e.g. Robinson, 1989). However, as is now clear, the electric field of the scattered wave field is itself effectively randomised by the randomly meandering contours of the plasma density in the ionosphere. This means that the electron motion driven by this electric field will already be random to some degree and does not have to rely entirely on the randomising effects of collisions with neutrals. Thus, the temperature of the electron gas is affected directly by the random component in the wave field and truly collisionless heating can arise for a sufficiently powerful pump wave.

Acknowledgement. The Editor in Chief thanks E. Mjølhus and T. Hagfors for their help in evaluating this paper.

\section{References}

Cohen, R. and Whitehead, J. D.: Radio-reflectivity detection of artificial modification of the ionospheric F-layer, J. Geophys. Res., 75, 6439-6445, 1970.

Dysthe, K. B., Mjølhus, E., Pécseli, H. L., and Rypdal, K.: A thermal oscillating two-stream instability, Phys. Fluids., 26, 146157, 1983.

Fialer, P. A.: Field-aligned scattering from a heated region of the ionosphere-Observations at HF and VHF, Radio Sci., 9, 923940, 1974.

Graham, K. N. and Fejer, J. A.: Anomalous radio wave absorption due to ionospheric heating effects, Radio Sci., 11, 1057-1063, 1976.

Gurevich, A. V.: Nonlinear phenomena in the ionosphere, Springer Verlag, Berlin, 1978.

Hagfors, T.: Electromagnetic wave propagation in a field-alignedstriated cold magnetoplasma with application to the ionosphere, J. Atmos. Terr. Phys., 46, 211-216, 1984.

Hedberg, Å., Derblom, H., Thidé, B., Kopka, H., and Stubbe, P.: Observations of HF backscatter associated with heating experiments at Troms $\varnothing$, Radio Sci., 18, 840-850, 1983.

Inhester, B., Das, A. C., and Fejer, J. A.: Generation of small-scale field-aligned irregularities in ionospheric heating experiments, J. Geophys. Res., 86, 9101-9106, 1981.

Jones, T. B., Robinson, T., Stubbe, P., and Kopka, H.: Frequency dependence of anomalous absorption caused by high power radio waves, J. Atmos. Terr. Phys., 46, 147-153, 1984.

Minkoff, J.: Radio frequency scattering from a heated ionospheric volume, 3, Cross section calculations, Radio Sci., 9, 997-1004, 1974.

Minkoff, J., Kugelman, P., and Weissman, I.: Radio frequency scattering from a heated ionospheric volume, 1, VHF/UHF fieldaligned and plasma-line backscatter measurements, Radio Sci., 9, 941-955, 1974.

Mjølhus, E.: Anomalous absorption and reflection in ionospheric radio modification experiments, J. Geophys. Res., 90, 42694279, 1985.

Robinson, T. R.: The heating of the high latitude ionosphere by high power radio waves, Phys. Rep., 179, 79-209, 1989.

Robinson, T. R., Stocker, A. J., Bond, G., Eglitis, P., Wright, D., and Jones, T. B.: O and X mode heating effects observed simultane- 
ously with the CUTLASS and EISCAT radars and low power HF diagnostics at Tromsø, Ann. Geophysicae, 15, 134-136, 1997.

Stix, T. H.: Waves in plasmas, Am. Inst. Phys., New York, 1992.

Stubbe, P., Kopka, H., Jones, T. B., and Robinson, T.: Wide band attenuation of radio waves caused by powerful HF waves: Satuaration and dependence on ionospheric variability, J. Geophys. Res., 87, 1551-1555, 1982.
Vaskov, V. V.: Dielectric constant perturbations caused by the conversion of an electromagnetic wave at small-scale plasma inhomogeneities, Sov. J. Plasma Phys. (Eng. Trans.), 14, 686-690, 1988.

Vaskov. V. V., and Gurevich, A. V.: Nonlinear resonant instability of a plasma in the field of an ordinary wave, Sov. Phys. JETP (Eng. Trans.), 42, 91-97, 1976. 